\section{Editor-in-Chief \\ Barbara McLain - (retired Prof.) \\ University of Hawaii, USA}

The Israeli Journal of Aquaculture (IJA) is an interdisciplinary journal that is dedicated to sharing new research and tested applications of aquaculture

The IJA is devoted to scholarly articles for improved aquaculture practices and related industries

The IJA is a peer-reviewed, open-access, electronic journal

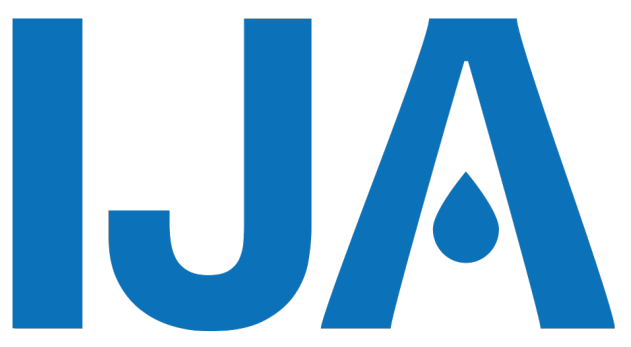

\section{The () Israeli Journal of Aquaculture}

An interdisciplinary online Open Access scientific journal

Published by the

\section{AquacultureHub}

A non-profit organization 501c3

http://www.aquaculturehub.org

in partnership with the

\section{University of Hawaii at Manoa} Library

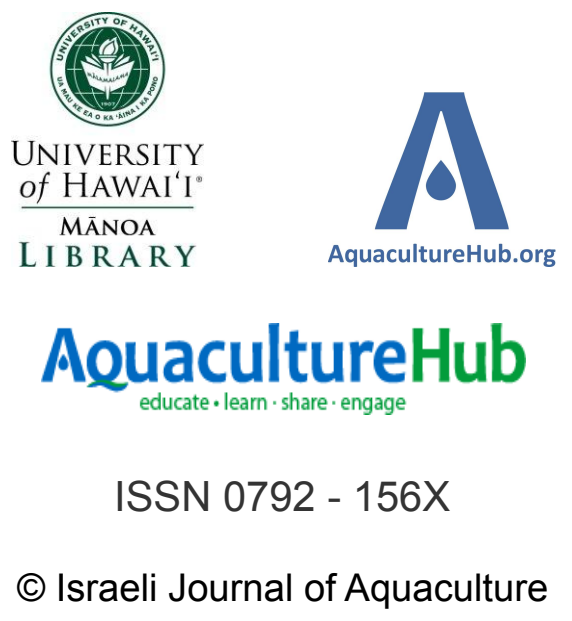



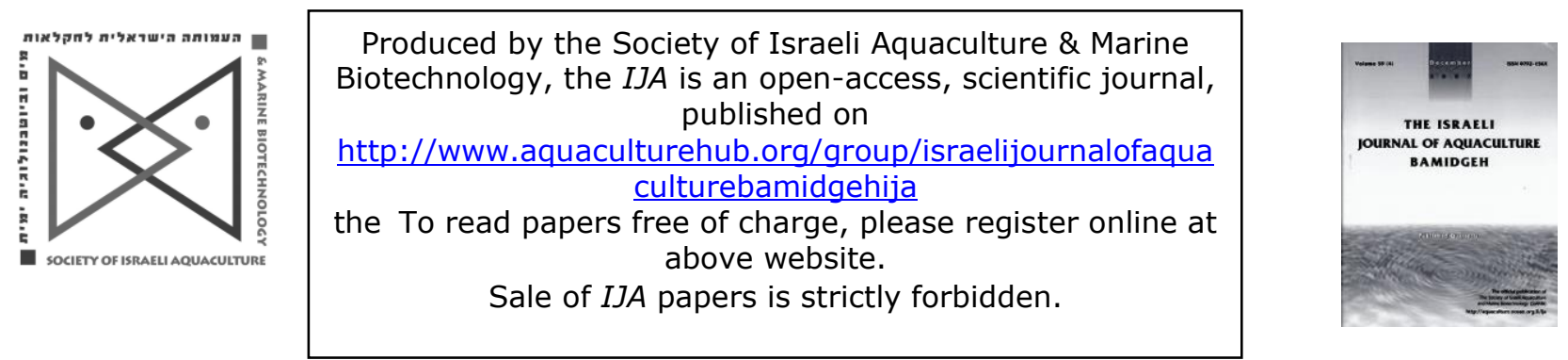

\title{
Effects of Dietary $\beta$-glucan Supplementation on Growth, Innate Immune, and Capacity Against Pathogen Streptococcus iniae of Juvenile Pompano (Trachinotus ovatus)
}

\author{
Do-Huu, $\mathbf{H}^{1,2^{*}}$, Nguyen, $\mathrm{TNH}^{1}$, and Tran, $\mathbf{V H}^{3}$ \\ ${ }^{1}$ Vietnam Academy of Science and Technology, Institute of Oceanography, \\ 01 Cau Da Street, Nha Trang City, Vietnam. \\ 2 Vietnam Academy of Science and Technology, Graduate University of \\ Science and Technology, 18 Hoang Quoc Viet Street, Cau Giay, Ha Noi, \\ Vietnam. \\ ${ }^{3}$ Nha Trang University, 02 Nguyen Dinh Chieu, Nha Trang, Vietnam.
}

Keywords: Pompano; Trachinotus ovatus; $\beta$-glucan; immune; Streptococcus iniae

\begin{abstract}
This study investigated the effect of dietary $\beta$-glucan on growth, immunity, and resistance against Streptococci in pompano $T$. ovatus. Pompano were fed a control diet, and diets which included $0.10 \%$ or $0.20 \%$ of $\beta$-glucan. After being fed the diets for 3-weeks, growth rate, survival, blood parameters, and immunity were examined, and the fish were challenged with Steptococcus aniae for 10 days. At the end of the feeding trial, RBC, WBC, WBC:RBC ratio, and lymphocytes significantly increased ( $P \leq 0.032$ ), while monocytes significantly decreased ( $P \leq$ 0.007 ) in fish fed $0.10-0.20 \%$ of $\beta$-glucan. Moreover, lysozyme was significantly higher ( $P \leq 0.003$ ) in fish fed $\beta$-glucan, while the phagocytic ratio was only significantly higher $(P=0.037)$ in fish fed $0.10 \% \beta$-glucan. Furthermore, fish fed $0.10-0.20 \% \beta$-glucan showed higher protection against $S$. iniae $(P \leq 0.002)$. Thus, a diet of $\beta$-glucan is recommended to improve non-specific immunity, growth in $T$. ovatus, and also to limit the adverse effects of $S$. aniae disease in this species.
\end{abstract}

* Corresponding author. Tel.: +84914258171, e-mail: dohuuhoang2002@yahoo.com 


\section{Introduction}

Pompano, Trachinotus ovatus (family Carangidae) is a highly valuable aquaculture species which is cultured in China, Singapore, Malaysia, and Vietnam (Chou and Lee, 1997; Do-Huu et al., 2016; Guo et al., 2018). This species has high economic value for the domestic and international market.

Pompano in aquaculture farms have occasionally endured outbreaks of diseases. At least, five pathogen species have been reported for Trachinotus blochii, including Streptococcus spp., Escherichia coli, Salmonella spp., Pseudomonas spp. and Vibrio spp. (Varol et al., 2014). Streptococcus are gram-positive bacteria, causing a systemic infection known as Streptococcosis (Eldar and Ghittino, 1999). Recently, S. iniae caused an outbreak in T. ovatus aquaculture in China (Guo et al., 2018). Streptococci including Streptococcus agalactiae, S. iniae, and S. dysgalactiae subsp. dysgalactiae were also isolated from pompano T. ovatus (Cai et al., 2016). Amongst the reported pathogens, Streptococcus species, S. agalactiae, and S. iniae have been mentioned more frequently than others (Cai et al., 2016). There is therefore an urgent need to find treatment for Streptococcosis in pompano.

In addition, Streptococcal disease is very difficult to control because at times it develops resistance to most chemicals and drugs (Klesius et al., 2008). Although a vaccine was successfully developed against streptococcal infection in fish, recent research reported that the Streptococcus iniae could re-appear even in vaccinated fish (Bachrach et al., 2001). In some cases, this pathogen was reported to be resistant to some antibiotics in pompano (Cai et al., 2016). The aim of this study was to try and find solutions that can enhance the sustainability of pompano aquaculture.

\section{Materials and Methods}

This study complies with the ethical guidelines and regulations concerning animal use for experimentation and was carried out with the approval of the Institute of Oceanography, Vietnam Academy of Science and Technology (VAST), Vietnam for animal experimentation.

Table 1. Diet formulation for experimental and control diets

\begin{tabular}{llll}
\hline Ingredients (\%) & $D 1$ & $D 2$ & $D 3$ \\
\hline Fish meal (62\% - Vietnam) & 44.60 & 44.60 & 44.60 \\
Gluten (wheat) & 21.10 & 21.10 & 21.10 \\
Soybean & 10.40 & 10.40 & 10.40 \\
Fish oil & 3.60 & 3.60 & 3.60 \\
Binder (inert) & 1.10 & 1.10 & 1.10 \\
Mineral premix & 1.50 & 1.50 & 1.50 \\
Vitamin premix & 1.50 & 1.50 & 1.50 \\
Corn starch & 16.20 & 16.10 & 16.00 \\
Macrogard ${ }^{\circledR}$ (ß-glucan) & 0 & 0.10 & 0.20 \\
TOTAL & $\mathbf{1 0 0}$ & $\mathbf{1 0 0}$ & $\mathbf{1 0 0}$ \\
\hline
\end{tabular}

\section{Diet preparation and feeding}

In this experiment, two doses of Marcrogard ${ }^{\circledR}(1$ and $2 \mathrm{~g} / \mathrm{kg})$ were incorporated in a basal diet formulated for pompano (crude protein: $48.6 \%$; crude lipid: $6.7 \%$ ). The formulation of a semi-purified diet for $\beta$-glucan and level of protein and lipid was referenced from other studies on pompano (Do-Huu et al., 2016). The supplemented levels of $\beta$-glucan were referenced from a previous study on pompano (Do-Huu et al., 2016; Do-Huu et al., 2018). All the ingredients were mixed, and water was slowly added to the ingredients to form a dough and passed through an extruder ( $2.5 \mathrm{~mm} \mathrm{die}$ ) to make pellets. The pellets were air dried and stored at $-4^{\circ} \mathrm{C}$ until use. During the experimental period, fish were fed to apparent satiation twice daily (at around 8:30 and $17: 30 \mathrm{~h})$.

\section{Experimental fish}

Healthy and disease-free pompano, T. ovatus of average body weight $30.43 \pm$ $0.21 \mathrm{~g}$ (average \pm SD) were obtained from a local hatchery and were acclimated for 2 
weeks in a composite tank of $5 \mathrm{~m}^{3}$ at the Marine Aquaculture Station at Institute of Oceanography, Nha Trang, Vietnam. During acclimation, fish were fed the basal diet at $4 \%$ of body weight and observed in satiation.

\section{Experimental tank and stocking}

After acclimation, twenty-four fish were stocked randomly into each 400 L-tank. A recirculation system with a flow rate of $400 \mathrm{~L}$ per minute was equipped in each tank. Fish were kept under natural photoperiod conditions and constant aeration throughout the trial. Water temperature was, $28-29.5^{\circ} \mathrm{C} ; \mathrm{NH}_{4}{ }^{+} / \mathrm{NH}_{3},<0.04 \mathrm{mg} / \mathrm{L} ; \mathrm{NO}_{2}{ }^{-}: 0.01 \mathrm{mg} / \mathrm{L}$ and $\mathrm{pH}$, 8.0-8.3.

\section{Experimental design}

The experiment was designed to evaluate non-specific immune, growth and mortality of fish fed different $\beta$-glucan levels and tolerance against Streptococcus iniae infection. There were six replicates for each diet treatments: a control basal diet, $0.10 \%$ and $0.20 \%$ of $\beta$-glucan supplementation diets. Fish were fed $\beta$-glucan supplemented diets or control diet for 3 weeks (stage 1) and then challenged against Streptococcus iniae and mortality was recorded for 10 days (stage 2). The fish were fed the same corresponding diet during the disease infection test.

\section{Sampling and data collection}

After stage one, (Day 21, end of feeding trial), all fish were weighed, and three fish per tank were collected for hematological determination and immune analysis. Prior to weighing and sampling, feed was not served for 24 hours. Fish were randomly collected and euthanized with $100 \mathrm{mg} / \mathrm{L}$ tricaine methanesulfonate (MS-222, Sigma, USA) and blood samples withdrawn with $1 \mathrm{~mL}$ syringes. A subdivision of the collected blood was placed into tubes with heparin as an anticoagulant to analyze the total erythrocyte and leucocyte count. The rest of the blood was placed in Eppendorf tubes and kept in ice, allowing the blood to clot for 2-4 h, followed by centrifuging for $10 \mathrm{~min}$. at $4000 \mathrm{~g}$ to collect serum. The serum was then stored at $-80^{\circ} \mathrm{C}$ for later lysozyme

analysis. After withdrawing blood, the head kidney was taken for analysis of phagocytic activity.

\section{Hematological assays}

Determination of leukocytes (WBC) and Erythrocytes (RBC) was conducted using a hemocytometer (Blaxhall and Daisley, 1973). Differential leukocyte counts including monocytes, lymphocytes, neutrophils, and eosinophils, were obtained by blood smears, air-dried, methanol fixed, and followed by staining in Diff-Quick ${ }^{\circledR}$. Leucocytes were classified relying on the morphology and counted under a microscope at a magnification of $1000 \times$.

\section{Isolation of leucocyte from head kidney}

Macrophages from head kidney were isolated following the method described by Secombes (1990). Briefly, the dissected head kidney was cut in small pieces and placed in RPMI-1640 medium supplemented with $100 \mathrm{IU} / \mathrm{mL}$ streptomycin, $100 \mathrm{IU} / \mathrm{mL}$ penicillin, $10 \mathrm{IU} / \mathrm{mL}$ heparin and $2 \%$ fetal calf serum (FCS), followed by grinding through a $100 \mu \mathrm{m}$ mesh. Then, $34 \% / 51 \%$ Percoll was added to the leucocyte cell suspension and centrifuged for $35 \mathrm{~min}$ at $1000 \mathrm{~g}\left(4^{\circ} \mathrm{C}\right)$. Subsequently, leucocyte cell interphase was collected and washed twice by using PBS, followed by centrifuging at $200 \mathrm{~g}$ (10 $\mathrm{min})$. Viable cell levels were determined using Trypan blue $(0.1 \%)$ and the cell density was checked by a hemocytometer. Cells were then suspended in L-15 medium with $0.1 \%$ FCS added to adjust the density to $1 \times 10^{7}$ cells $/ \mathrm{mL}$ for analysis.

Phagocytic activity

The phagocytosis assay of pompano leukocytes was performed according to the method of Siwicki et al. (1994) with a slight modification. The $100 \mu \mathrm{L}$ cells of head kidney leucocytes $\left(1 \times 10^{7}\right.$ cells $/ \mathrm{mL}$ ) were placed in the sterile slide (3 replicates slide for each fish) for $\sim 30 \mathrm{~min}$ (at $25^{\circ} \mathrm{C}$ ). Then, $100 \mu \mathrm{L}$ zymosan suspension (Sigma) $1 \times 10^{8} \mathrm{cells} / \mathrm{mL}$ were added, mixed then incubated for $1 \mathrm{~h}$ at $25^{\circ} \mathrm{C}$. The slide was washed by PBS to remove unattached cells. After drying at room temperature, the slide was fixed with methanol and stained with $1 \mathrm{~mL}$ Congo Red for $5 \mathrm{~min}$. A total of about five hundred phagocytes per slide were observed under a microscope under oil immersion at $1000 \times$ 
magnification. The phagocytic activity was estimated as the phagocytic ratio $(P R, \%)=$ $\mathrm{nr}$. of phagocytic cells with engulfed zymosan/nr. of phagocytic cells (Sahoo and Mukherjee, 2001).

Serum lysozyme activity

To determine lysozyme, $25 \mu \mathrm{L}$ of collected serum was microplated with 3 well replicates for each, and PBS was used as blank. Then, hen egg lysozyme dilutions of (0 $20 \mu \mathrm{g} / \mathrm{mL}$; Sigma-Aldrich) were used to create a standard curve. Then, $175 \mu \mathrm{L}$ of the Micrococcus lysodeikticus $(0.75 \mathrm{mg} / \mathrm{mL})$. Next, the microplate was mixed at $25^{\circ} \mathrm{C}$. The difference in turbidity was recorded by a microwell plate reader $440 \mathrm{~nm}$ at $15 \mathrm{~min}$ and 30 min (Ellis, 1990).

Streptococcus challenge trial

The Streptococcus iniae bacteria were provided by Nha Trang University. Three weeks post diet feeding, eighteen fish from each diet treatment were randomly collected and injected with Streptococcus iniae (stage 2). Each fish was injected intraperitoneally with $100 \mu \mathrm{L}$ suspension of Streptococcus $\left(1.5 \times 10^{4} \mathrm{cfu} / \mathrm{fish}\right)$ in PBS using a $0.3 \mathrm{~mL}$ insulin syringe. One treatment was held in three $400 \mathrm{~L}$-tanks as replicates. The injection dose of bacteria is a lethal dose $50 \%$ ( $L D_{50}$ ) as described in below. For negative control, ten fish from the group of fish fed the control diet were collected and injected with 100 $\mu \mathrm{L}$ saline water, and these fish were kept in separately to avoid cross infection. The fish in each diet treatment were given the same assigned diets during the challenge test with adjustment for satiation by observation. Fish mortality was recorded twice a day for 10 days. It was assumed that the longer a fish survived, the greater was its resistance to Streptococcus iniae infection.

Determination of lethal dose $50 \%$ (LD50)

The lethal dose, $L D_{50}$ at ten days was determined by intraperitoneal injecting 100 $\mu \mathrm{L}$ inoculum per fish with graded doses of Streptococcus iniae $\left(10^{2}, 10^{3}, 10^{4}, 10^{5}, 10^{6}\right.$ and $10^{7} \mathrm{cfu} / \mathrm{fish}$ ) at $10 \mathrm{fish}$ for each dose. The dose of $1.5 \times 10^{4} \mathrm{cfu} /$ fish was selected for the challenge test for ten days in this experiment.

Data calculation and statistical analysis

Specific Growth rate (SGR \%/d), survival rate, and relative percent survival (RPS) was calculated as follows:

$\mathrm{SGR}=100 \times[\mathrm{Ln}(\mathrm{Wt})-\mathrm{Ln}(\mathrm{Wo})] /$ days; $\mathrm{S}=100 \times(\mathrm{nt} / \mathrm{no}) ; \mathrm{RPS}=100 \times(1-\mathrm{Md} /$ Mo) (Amend, 1981),

where $\mathrm{Wt}$ and Wo were the weight of the fish at time $\mathrm{t}$; and at the commencement; S: Survival rate (\%), nt and no were the number of the fish at time $t$ and at the commencement, $\mathrm{Md}$ and Mo were the percentage mortalities of fish on $\beta$ glucan diets and control diet treatments, respectively.

ANOVA was applied to compare average growth and survival value among diet treatments. Data were tested before analysis. The post hoc test (LSD) was used where significance was detected. Cumulative survival curves of Streptococcus infected fish fed dietary $\beta$-glucan levels compared to fish fed control diet were determined by KaplanMeier followed by log-rank test. Relative risk, hazard ratio between diets were also analyzed. $\mathrm{P}<0.05$ was considered significant in each case. All statistics were performed using SPSS 18.

\section{Results}

Growth performance of pompano fed dietary $\beta$-glucan

Initial mean weight of pompano was uniform among the treatments, with no significant difference (ANOVA, $\mathrm{P}=0.842$ ). Fish weight reached over $35 \mathrm{~g}$ at the end of the feeding trial (Day 21). After 21-days of diet feeding, the specific growth rate (SGR) of pompano fed $0.10 \%(D 2)$ was higher than SGR of fish fed control diet $(P=0.010)$. However, there was no significant difference $(P \geq 0.221)$ either between SGR of fish fed D1 and fish fed D3 or between fish fed diet D2 and D3. (Fig. 1) 


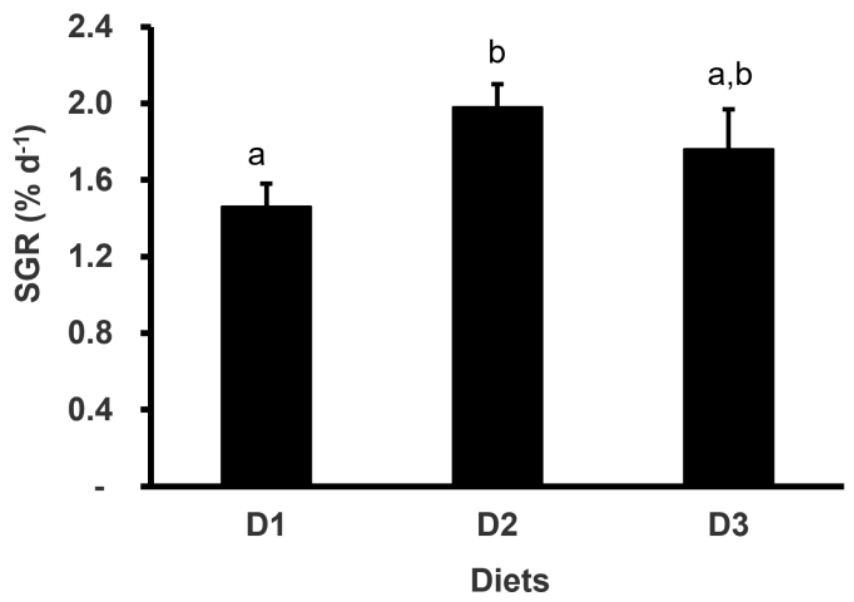

Fig. 1. Growth performance of pompano fed dietary $\beta$ glucan supplementation. Data are presented as the means \pm SD. Different letters indicate significant difference between treatments.

Survival rate and relative survival rate (RSR) of pompano

At the end of the feeding trial (Day 21), survival rates of fish ranged from $86.01 \%$ (in the fish fed control diet) to $94.63 \%$ (in the fish fed diet with $0.10 \% \beta$-glucan, D2) (Fig. 2). Survival rate was highest in the pompano group fed the diet supplemented with $0.10 \% \beta$-glucan and was significantly higher than the survival of the control group of pompano $(P=0.001)$. However, the survival of fish fed the diet supplemented with $0.20 \% \beta$-glucan (D3) did not significantly differ from the survival rates of the control group of fish or fish fed diet with $0.10 \% \beta$-glucan (D2) ( $P \geq 0.120$ ). Relative survival percentages were $60.6 \%$ and $54.6 \%$ for groups of fish fed diets supplemented with $0.10 \%$ (D2) and $0.20 \% \beta$-glucan (D3), respectively (Fig. 2).

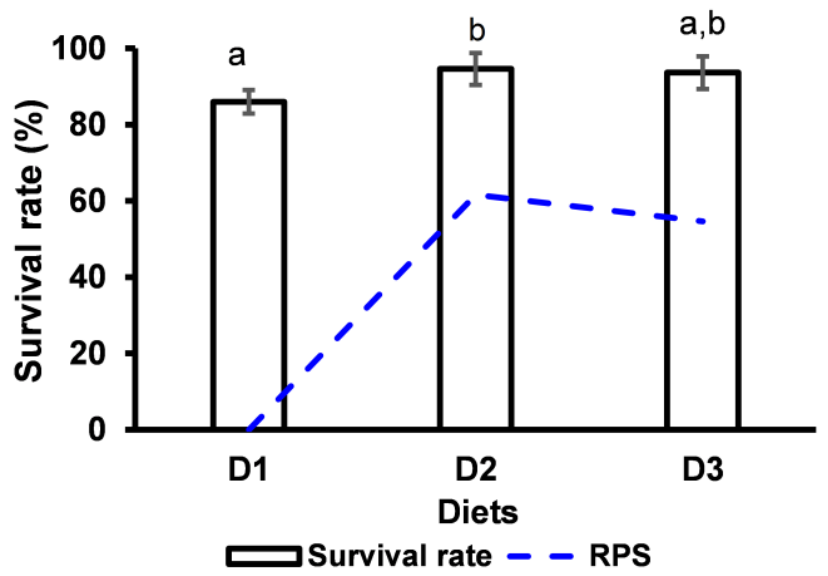

Fig. 2. Survival rate and relative percentage survival (RPS) of pompano fed different levels of dietary $\beta$ glucan. Data are presented as the means \pm SD. Different letters indicate significant difference between treatments.

Hematological parameters of pompano fed dietary $\beta$-glucan supplementation

There was a significant difference in the hematological index between the dietary treatments. White blood (WBC) and red blood cells (RBC) of pompano fed both levels of Macrogard ${ }^{\circledR}$ were higher than the fish fed control diet $(P \leq 0.004)$, but there were no differences ( $P \geq 0.249$ ) between them (Table 2). Ratios of WBC:RBC were also higher in fish fed diet supplemented with $0.10 \%$ (D2) and $0.20 \% \beta$-glucan (D3) compared to fish fed control diet $(P \leq 0.012)$, but no significance $(P \geq 0.564)$ in the WBC:RBC ratio among the fish fed two levels of $\beta$-glucan (Table 2 ). The leukocytes, lymphocytes, and monocytes altered significantly in fish fed dietary $\beta$-glucan supplementations compared to fish fed control. Pompano fed the diet with $0.10 \%$ (D2) and $0.20 \% \beta$-glucan (D3) inclusion showed significantly higher proportions of lymphocytes, in comparison to fish fed control $(P \leq 0.032)$. In contrast, monocytes were significantly reduced in fish fed $\beta$ glucan in the diet $(P \leq 0.007)$. However, lymphocytes and monocytes were not different between fish fed diets supplemented with $0.10 \%$ (D2) and $0.20 \% \beta$-glucan (D3) $(P \geq$ 0.363). In addition, dietary $\beta$-glucan showed no significant influence on neutrophil and 
basophil cell counts $(P \geq 0.104)$. However, eosinophil was significantly lower $(P=0.022)$ in fish fed $0.10 \%$ (D2) compared to fish fed control (Table 2).

Table 2. Hematological parameters of pompano after 3-weeks of diet feeding. Different letters indicate significant difference between treatments at each time point.

\begin{tabular}{llll}
\hline & & \multicolumn{2}{c}{ Diets } \\
& $D 1$ & $D 2$ & $D 3$ \\
\hline RBC $\left(\times 10^{6} \mathrm{~mm}^{-3}\right)$ & $4.41 \pm 0.11^{\mathrm{a}}$ & $5.22 \pm 0.08^{\mathrm{b}}$ & $5.16 \pm 0.17^{\mathrm{b}}$ \\
WBC $\left(\times 10^{5} \mathrm{~mm}^{-3}\right)$ & $0.88 \pm 0.05^{\mathrm{a}}$ & $1.23 \pm 0.07^{\mathrm{b}}$ & $1.23 \pm 0.09^{\mathrm{b}}$ \\
Monocyte $(\%)$ & $19.90 \pm 1.68^{\mathrm{a}}$ & $14.05 \pm 1.76^{\mathrm{b}}$ & $12.51 \pm 0.49^{\mathrm{b}}$ \\
Lymphocyte (\%) & $66.60 \pm 1.96^{\mathrm{a}}$ & $73.49 \pm 1.12^{\mathrm{b}}$ & $73.77 \pm 1.45^{\mathrm{b}}$ \\
Neutrophil (\%) & $9.81 \pm 0.62$ & $10.10 \pm 1.01$ & $10.82 \pm 1.13$ \\
Eosinophil (\%) & $2.76 \pm 0.69^{\mathrm{a}}$ & $0.91 \pm 0.32^{\mathrm{b}}$ & $1.75 \pm 0.54^{\mathrm{ab}}$ \\
Basophil (\%) & $0.95 \pm 0.66$ & $1.45 \pm 0.40$ & $1.15 \pm 0.56$ \\
WBC : RBC (\%) & $0.20 \pm 0.02^{\mathrm{a}}$ & $0.28 \pm 0.01^{\mathrm{b}}$ & $0.26 \pm 0.02^{\mathrm{b}}$ \\
\hline
\end{tabular}

Phagocytic ratio of pompano

There were significant increases in the phagocytic ratios (PR) of pompano fed the diet D2 $(0.10 \% \beta$-glucan) compared to PR value in fish fed control diet (D1) $(P=0.037)$, while no statistical significance was observed in PR of fish fed diet D3 (0.20\% $\beta$-glucan) compared to control $(P=0.059)$. Also, the $P R$ values were not significantly different $(P=0.531)$ between fish fed diets D2 and D3. (Fig. 3).

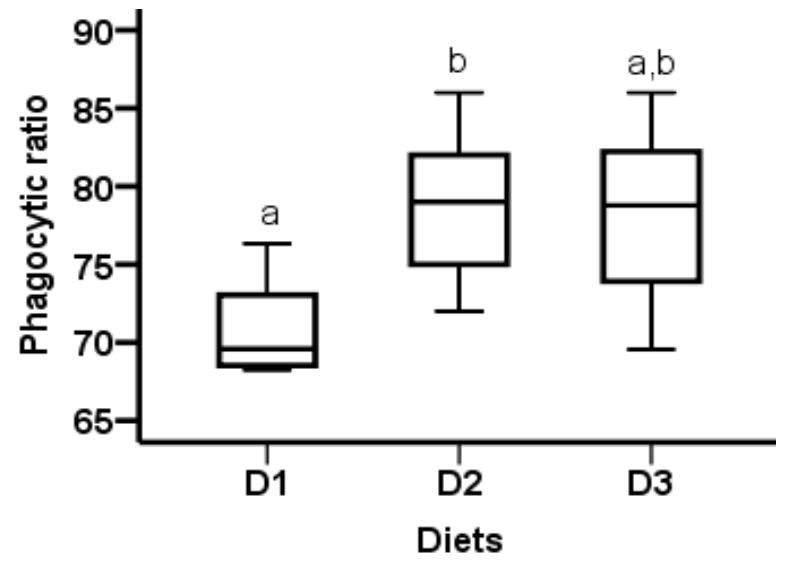

Fig. 3. The phagocytic ratio $(\mathrm{PR})$ in pompano fed different levels of $\beta$-glucan. Data are presented as the means \pm SD. Different letters indicate significant difference between treatments.

Lysozyme activity of pompano fed dietary $\beta$-glucan

Post-3-weeks of diet feeding, dietary $\beta$-glucan supplementation significantly enhanced serum lysozyme activity in pompano. The highest level of lysozyme was in fish fed diet D2, followed by the fish fed diet D3, which were both significantly higher than lysozyme activity in fish fed the control $(P \leq 0.003)$. However, no significant difference $(P=0.053)$ was found in lysozyme activities of fish fed diets $D 2(0.10 \% \beta$-glucan $)$ and D3 (0.20\% $\beta$-glucan). (Fig. 4)

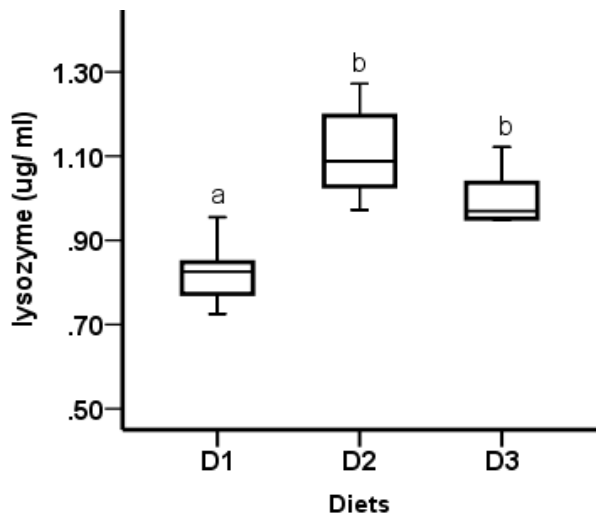

Fig. 4. Serum lysozyme activity of pompano fed different levels of dietary $\beta$ glucan supplementation. Data are presented as the means \pm SD.

Different letters indicate significant difference between treatments. 
Cumulative mortalities, relative risk, and hazard ratio of pompano challenged with Streptococcus iniae

After 21 days of feeding with $\beta$-glucan supplemented diets, the pathogen challenge test was performed for 10 days. The accumulative survival rate was determined on the $10^{\text {th }}$ day and is presented in Fig. 5. During the infection period, there were no mortalities in the negative control group. After challenging with Streptococcus, the first mortality was recorded after 2 days in the control group, while in fish fed diet $0.10 \%$ and $0.20 \% \beta$-glucan the first mortality occurred on day 3 , respectively. After 10 days, the highest survival rate was observed in fish fed $0.10 \%$, followed by fish fed $0.20 \% \beta$-glucan inclusion in the diet, which were both significantly higher $(P \leq 0.002)$ than fish fed control diet (D0.00). The survival rates of fish fed diets supplemented with $0.10 \%$ and $0.20 \% \beta$-glucan did not significantly differ $(P=0.850)$ during the challenge period (Fig. 5). When exposed to pathogen, the hazard ratio of fish fed diets supplementation of $0.1 \%$ and $0.2 \%$ were 0.216 (95\% CI: 0.0873 to 0.5327 ) and 0.223 (95\% CI: 0.0912 to 0.5453 ), respectively.

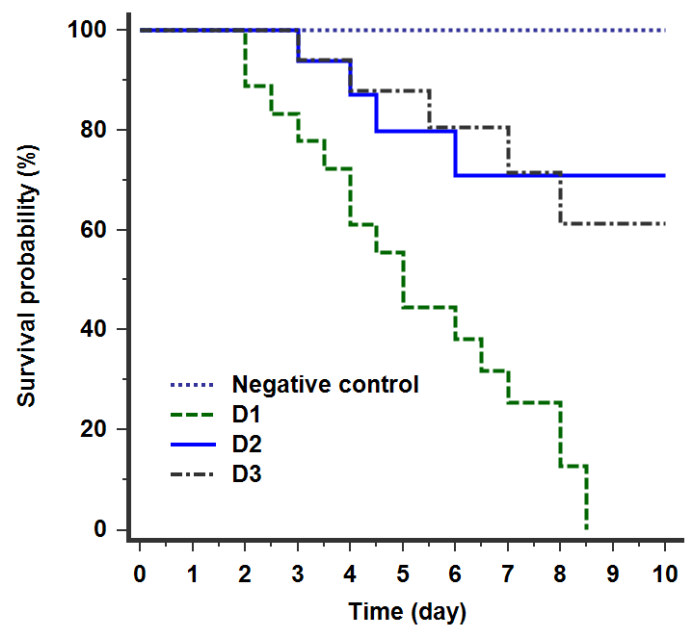

Fig. 5. Survival curves of juvenile pompano fed $\beta$-glucan diets and challenged with Streptococcus

\section{Discussion}

To our knowledge, this is the first finding showing the efficiency of dietary $\beta$-glucan supplementation on boosting the resistance of pompano against Streptococcus iniae pathogen infection. Also, the growth and hematological index and immune response were higher in fish fed $\beta$-glucan diet.

In line with the previous study (Do-Huu et al., 2016), the present study confirmed that the growth and survival of pompano were significantly improved when their diet was supplemented with $0.10 \% \beta$-glucan. This is also in agreement with another study which revealed that dietary $\beta$-glucan could improve growth in aquaculture species including red sea bream Pagrus major (Dawood et al., 2017). In contrast, $\beta$-glucan has shown no effects on the growth of European sea bass Dicentrarchus labrax (Bagni et al., 2005). Possible factors are the concentration of $\beta$-glucan administration in the diet, speciesspecific response, and experimental conditions as well as the administration time (DoHuu et al., 2016). In the present study, juvenile pompano grew rapidly and the significant differences were detected between diet treatments after 21-days of feeding. Research reveals that fast growth rate may result from the energetic benefits obtained through $\beta$-glucan administration (Ai et al., 2007), manipulation of intestinal microbiota (Do-Huu et al., 2016), or digestive enzymes produced by beneficial bacteria (Wu et al., 2014).

In this study, total leucocyte count increased in the fish fed $\beta$-glucan supplemented diets. Similarly to our previous study (Do-Huu et al., 2016), the present study showed that lymphocyte and WBC increased significantly in pompano, T. ovatus, supplied $0.10 \% \beta$-glucan in the diet. In agreement with our results, lymphocyte and WBC 
increased in Persian sturgeon fish Acipenser persicu fed dietary $\beta$-glucan (Aramli et al., 2015).

In the innate immune system, fish blood lysozyme was also proposed as a valuable indicator to reflect the bio-defense level and in evaluating the influences of immunostimulants (Siwicki and Anderson, 1993). Also, lysozyme is a significant defense enzyme, which exists in the blood and lymphoid tissues of fish. The enzyme plays a vital role in fish health and is one of the most important natural resistances in fish protection against microbial invasion (Saurabh and Sahoo, 2008). It was also reported that $\beta-$ glucan administration resulted in increase in serum lysozyme, phagocytic activity, and induced macrophages hyperplasia, enhanced state of host defense against disease (Kokoshis et al., 1978). Both lysozyme and IgM activities can reflect the immune function of fish in alternative environments or nutritional conditions (Lin and Shiau, 2003). Additionally, $\beta$-glucan administration was reported to stimulate lysozyme, phagocytic activity, antibody production and respiratory bursts of many species such as sea bass Dicentrarchus labrax (Bagni et al., 2005). Furthermore, dietary $\beta$-glucan supplementation significantly enhanced lysozyme activity of large yellow croaker Pseudosciaena crocea (Ai et al., 2007). The result of this study is in line with other findings that increasing lysozyme by dietary $\beta$-glucan suggesting that the $\beta$-glucan might contain some bioactive polysaccharide involving in regulating the host immune response (Kokoshis et al., 1978).

Studies have indicated that streptococcal diseases are very difficult to control because they are resistant or have become resistant to most chemicals and drugs (Cai et al., 2016). In addition, although vaccine was applied to treat streptococcal infection in fish, the study has shown that the Streptococcus pathogen could recover again even in vaccinated fish (Bachrach et al., 2001). The treatment for streptococcal disease in pompano is still not fully determined (Cai et al., 2016). Results in this study suggest that $\beta$-glucan diet could stimulate some degree of immunity in pompano. Dietary $\beta$-glucan supplementation at $0.10-0.20 \%$ in the diet of pompano was significantly associated infection of $S$. aniae risk, presenting a significantly lower risk when challenged against Streptococcus in comparison to fish fed basal diet. Therefore, the present findings can be beneficial for farmers by reducing the use of antibiotics in aquaculture and improving sustainability. However, further research should be conducted on different sized fish, using different doses, and adjusting administration periods.

Research has also revealed that dietary $\beta$-glucan administration increases resistance to other disease infection in aquaculture fish. In fact, dietary $\beta$-glucan has shown to significantly increase survival of Pseudosciaena crocea challenged with Vibrio harveyi (Ai et al., 2007) and in Labeo rohita fingerlings against Edwardsiella tarda and Aeromonas hydrophila infection (Misra et al., 2006). In line with these findings, our results show that dietary $\beta$-glucan promoted higher resistance of pompano to $S$. iniae pathogen infection together with elevated immune response.

In conclusion, the present findings confirm the supplementation of $\beta$-glucan in the diet is beneficial in boosting non-specific immunity, growth performance, survival rate, and tolerance to Streptococcus iniae infection of pompano $T$. ovatus. The addition of $0.10 \%$ of $\beta$-glucan to the pompano diet is recommended to boost disease resistance, immunity, and growth performance. However, further potential benefits of $\beta$-glucan application such as boosted resilience other infectious pathogens should be explored. In addition, the influence of dietary $\beta$-glucan on different life stages such as larval and broodstock may be tested experimentally.

\section{Acknowledgements}

This research was funded by the Vietnam National Foundation for Science and Technology Development (NAFOSTED) under grant number: 106-NN.05-2019.04. We particularly would like to thank Dr. Oliveira F.R.F. and Biorigin for generously providing MacroGard ${ }^{\circledR}$. The authors would like to express special thanks to Dr. Nguyen Thi Thanh Thuy, Dr. Huynh Minh Sang, Nguyen Thi Kim Bich, Cao Van Nguyen, Ho Son Lam and Tran Le Khanh Ha for their great help. 


\section{References}

Ai Q., K. Mai, L. Zhang, B. Tan, W. Zhang, W. Xu and H. Li, 2007. Effects of dietary $\beta-1,3$ glucan on innate immune response of large yellow croaker, Pseudosciaena crocea. Fish Shellfish Immunol., 22: 394-402.

Amend D.F., 1981. Potency testing of fish vaccines. Dev. Biol. Stand., 49: 447-454.

Aramli M.S., B. Kamangar and R.M. Nazari, 2015. Effects of dietary $\beta$-glucan on the growth and innate immune response of juvenile Persian sturgeon, Acipenser persicus. Fish Shellfish Immunol., 47: 606-610.

Bachrach G., A. Zlotkin, A. Hurvitz, D.L. Evans and A. Eldar, 2001. Recovery of Streptococcus iniae from Diseased Fish Previously Vaccinated with a Streptococcus Vaccine. Appl. Environ. Microbiol., 67: 3756-3758.

Bagni M., N. Romano, M.G. Finoia, L. Abelli, G. Scapigliati, P.G. Tiscar, M. Sarti and G. Marino, 2005. Short- and long-term effects of a dietary yeast beta-glucan (Macrogard) and alginic acid (Ergosan) preparation on immune response in sea bass (Dicentrarchus labrax). Fish Shellfish Immunol., 18: 311-325.

Blaxhall P.C. and K.W. Daisley, 1973. Routine haematological methods for use with fish blood. J. Fish Biol., 5: 771-781.

Cai X.H., Y.H. Peng, Z.C. Wang, T. Huang, X.Y. Xiong, Y.C. Huang, B. Wang, L.W. $\mathbf{X u}$ and Z.H. Wu, 2016. Characterization and identification of streptococci from golden pompano in China. Dis. Aquat. Org., 119: 207-217.

Chou R. and H.B. Lee, 1997. Commercial marine fish farming in Singapore. Aquac. Res., 28: 767-776.

Dawood M.A.O., S. Koshio, M. Ishikawa, S. Yokoyama, M.F. El Basuini, M.S. Hossain, T.H. Nhu, A.S. Moss, S. Dossou and H. Wei, 2017. Dietary supplementation of $\beta$-glucan improves growth performance, the innate immune response and stress resistance of red sea bream, Pagrus major. Aquac. Nutr., 23: 148-159.

Do-Huu H., H.M. Sang and N.T. Thanh Thuy, 2016. Dietary $\beta$-glucan improved growth performance, Vibrio counts, haematological parameters and stress resistance of pompano fish, Trachinotus ovatus Linnaeus, 1758. Fish Shellfish Immunol., 54: 402-410.

Do-Huu H., S.L. Ho and V.N. Cao, 2018. Efficiency of Dietary $\beta$-glucan Supplementationon Growth, Body Composition, Feed, and Nutrient Utilization in Juveniles of Pompano Fish (Trachinotus ovatus, Linnaeus, 1758). Isr. J. Aquacult-Bamidgeh, AquacultureHub, 70: 1518; 13.

Eldar A. and C. Ghittino, 1999. Lactococcus garvieae and Streptococcus iniae infections in rainbow trout Oncorhynchus mykiss: similar, but different diseases. Dis. Aquat. Organ., 36: 227-231.

Ellis A., 1990. Lysozyme assays. In: Stolen JS, Fletcher TC, Anderson DP, Robertsen BS, Va $n$ Muiswinkel WB, editors. Techniques in fish immunology. Fair Haven, NJ: SOS Publications. p. 101e3.

Guo S., Z. Mo, Z. Wang, J. Xu, Y. Li, X. Dan and A. Li, 2018. Isolation and pathogenicity of Streptococcus iniae in offshore cage-cultured Trachinotus ovatus in China. Aquaculture, 492: 247-252.

Klesius P.H., C.A. Shoemaker and J.J. Evans, 2008. Streptococcus: A worldwide fish health problem. 8th International Symposium on Tilapia in Aquaculture. Cairo, 83-107.

Kokoshis P., D. Williams, J. Cook and N. Di Luzio, 1978. Increased resistance to Staphylococcus aureus infection and enhancement in serum lysozyme activity by glucan. Science, 199: 1340-1342.

Lin Y.-H. and S.-Y. Shiau, 2003. Dietary lipid requirement of grouper, Epinephelus malabaricus, and effects on immune responses. Aquaculture, 225: 243-250.

Misra C.K., B.K. Das, S.C. Mukherjee and P. Pattnaik, 2006. Effect of multiple injections of $\beta$-glucan on non-specific immune response and disease resistance in Labeo rohita fingerlings. Fish Shellfish Immunol., 20: 305-319.

Sahoo P.K. and S.C. Mukherjee, 2001. Effect of dietary $\beta-1,3$ glucan on immune responses and disease resistance of healthy and aflatoxin B1-induced immunocompromised rohu (Labeo rohita Hamilton). Fish Shellfish Immunol., 11: 683695. 
Saurabh S. and P.K. Sahoo, 2008. Lysozyme: an important defence molecule of fish innate immune system. Aquac. Res., 39: 223-239.

Secombes C.J., 1990. Isolation of salmonid macrophages and analysis of their killing activity. In: Techniques in Fish Immunology (ed. by J.S. Stolen, T.C. Fletcher, D.P. Anderson, B.S. Roberson \& W.B.V. Muiswinkel), pp. 137-154. SOS Publications, Fair Haven, NJ.

Siwicki A.K. and D.P. Anderson, 1993. Nonspecific defense mechanisms assay in fish: II. potential killing activity of neutrophils and macrophages, lysozyme activity in serum and organs and total immunoglobulin level in serum. Fish Disease Diagnosis and Prevention Methods. Olsztyn, Poland. 105-111.

Siwicki A.K., D.P. Anderson and G.L. Rumsey, 1994. Dietary intake of immunostimulants by rainbow trout affects non-specific immunity and protection against furunculosis. Vet. Immunol. Immunopathol., 41: 125-139.

Varol E., H.A. Bas, F. Aksoy, H. Ari and M. Ozaydin, 2014. Relationship Between Neutrophil-Lymphocyte Ratio and Isolated Low High-Density Lipoprotein Cholesterol. Angiology, 65: 630-633.

Wu Z.-X., Y.-M. Yu, X. Chen, H. Liu, J.-F. Yuan, Y. Shi and X.-X. Chen, 2014. Effect of prebiotic konjac mannanoligosaccharide on growth performances, intestinal microflora, and digestive enzyme activities in yellow catfish, Pelteobagrus fulvidraco. Fish Physiol. Biochem., 40: 763-771. 